\title{
Crystallographic Reconstruction Methods to Study Phase Transformations by EBSD
}

C. Cayron*

*CEA, Laboratory for Innovation in New Energy (LITEN), Minatec, 17 rue des Martyrs, 38054 Grenoble Cedex 9, France

EBSD is powerful technique to measure the orientations of the grains in crystalline materials with a spatial resolution better than $10 \mathrm{~nm}$ with a FEG-SEM. At the beginning of its automation, in the 1990s, this technique was mainly used to map the textures in deformed materials. It is also now an important tool to study phase transformations thanks to recent progresses such as fast cameras and new SEM in-situ heating and deformation modules that allow direct mapping of the transformations, FIB sectioning that allows 3D imaging of the morphologies of the product phases linked to their orientations, and advanced computer treatments of the crystallographic data (subject of this talk).

Crystallography of phase transformations is based on group theory [1-2]. The crystallographic information of any structural transformation can be encoded in a groupoid composition table [2]. Cycle of phase transformations is still an open subject and algebraic/crystallographic theories have been developed "only" for multiple twinning in cubic materials. Strong links were shown between cycles of transitions, fractals, groupoids and string theories [3, 4]. We have written a computer program ARPGE [5] that use these theories to automatically treat the EBSD maps and perform crystallographic reconstructions: (a) reconstruction of the parent grains without the help of possible retained parent phase in phase transition materials such as iron, steels, titanium and zirconium alloys etc., and (b) recognition of the twin related domains (TRDs) in low staking fault cubic materials such as copper, silicon, etc.

New and important statistical microstructural parameters are now easily accessible:

(a) In phase transition materials: the sizes and textures of parent grains and the distribution of operators between variants (a high frequency of an operator means a strong variant selection mechanism). See an example in Fig. 1 of the reconstruction of the austenitic grains in a bainitic steel with Kurdjumov-Sachs orientations.

(b) In multiply twinned materials: the size of the TRDs, the twinning orders and the distribution of the $\sum 3^{\mathrm{n}}$ operators. See an example in Fig. 2 of the reconstruction of the TRDs in a copper thin film with an example of the fractal representation of the twinning chain in one of the TRDs of the map.

References

[1] U. Dahmen, Phase Transformations, Crystallographic Aspects in Encyclopedia of Physical Science and Technology. Academic Press, San Diego, 1987, pp 319-354.

[1] C. Cayron, Acta Cryst. A62 (2006) 21.

[2] C. Cayron, Acta Cryst. A63 (2007) 11.

[3] B.W. Reed et al, Acta Cryst. A60 (2004) 263.

[4] C. Cayron, J. Appl. Cryst. 40 (2007), 1183.

[5] PH Jouneau, B. Artaud and L. Briottet are acknowledged for their support during the developments of ARPGE. 


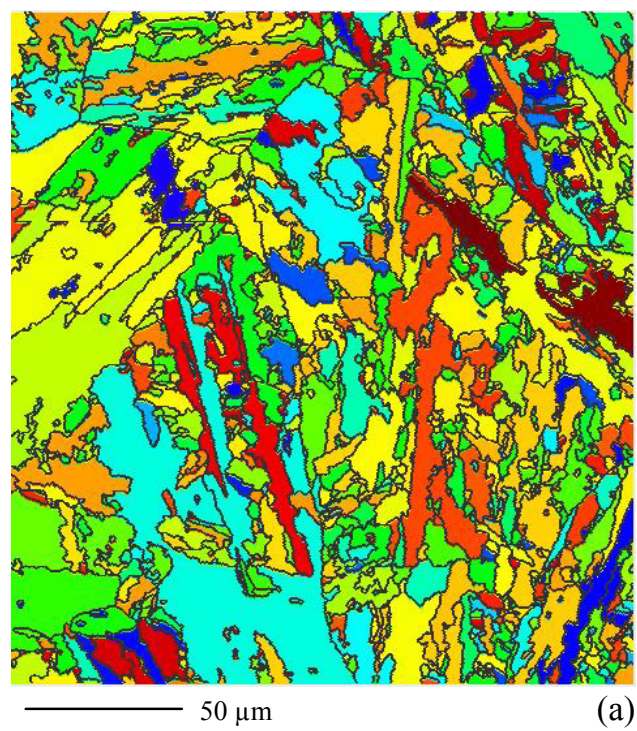

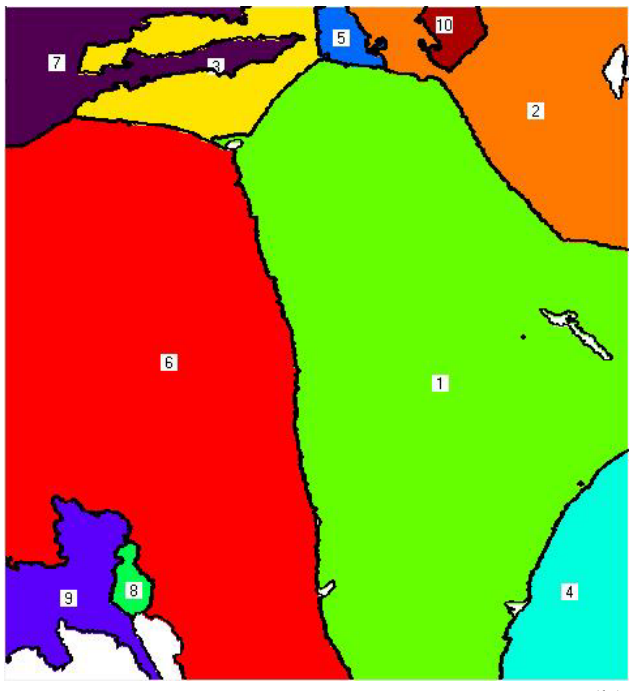

(b)

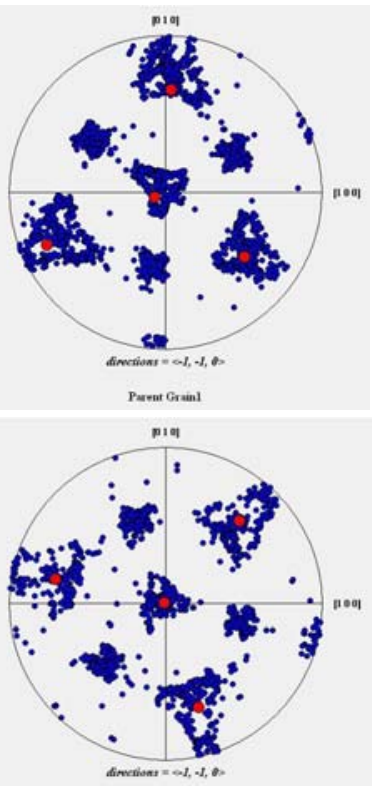

Pusmu Grains (c)

(d)

FIG. 1. Reconstruction of the austenitic grains in a bainitic steel with ARPGE (assuming a KS OR): (a) Orientation map of the bainitic grains. (b) Reconstructed austenitic grains (random colors). Calculated orientations of grains 1 (red) and 6 (green) are given in the pole figures (c) and (d). The experimental $<110>$ bainitic directions and the calculated $<111>$ austenitic directions are represented by the blue and red disks respectively.
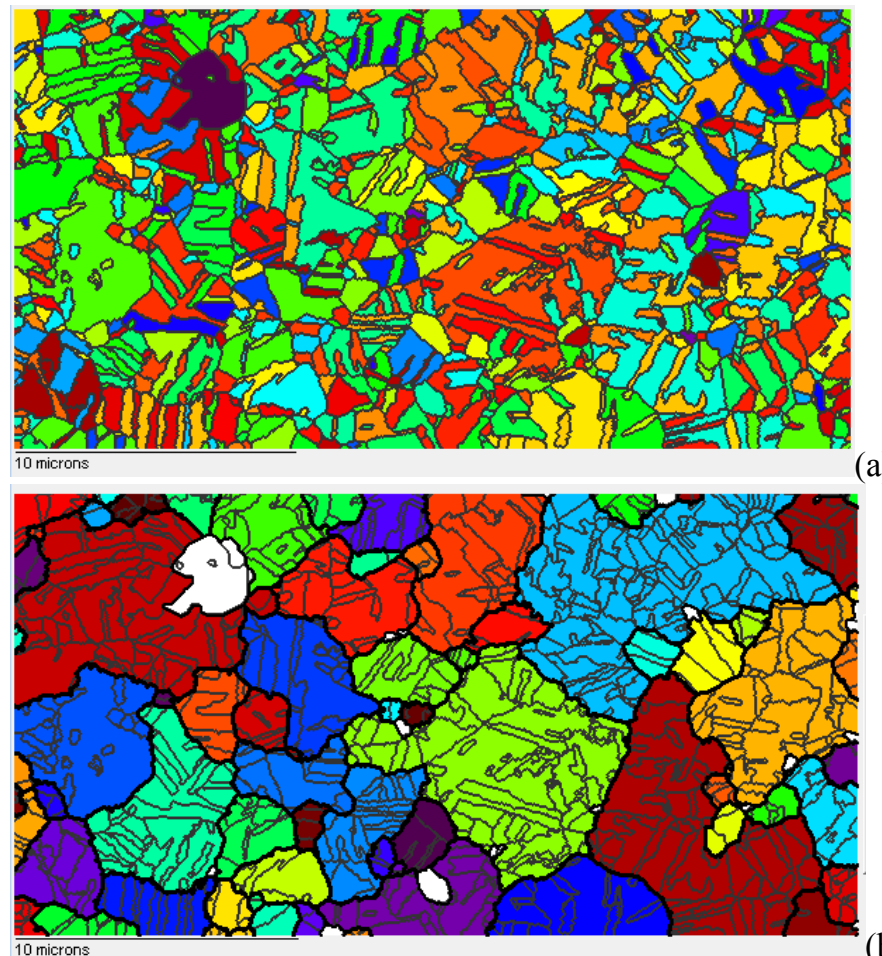

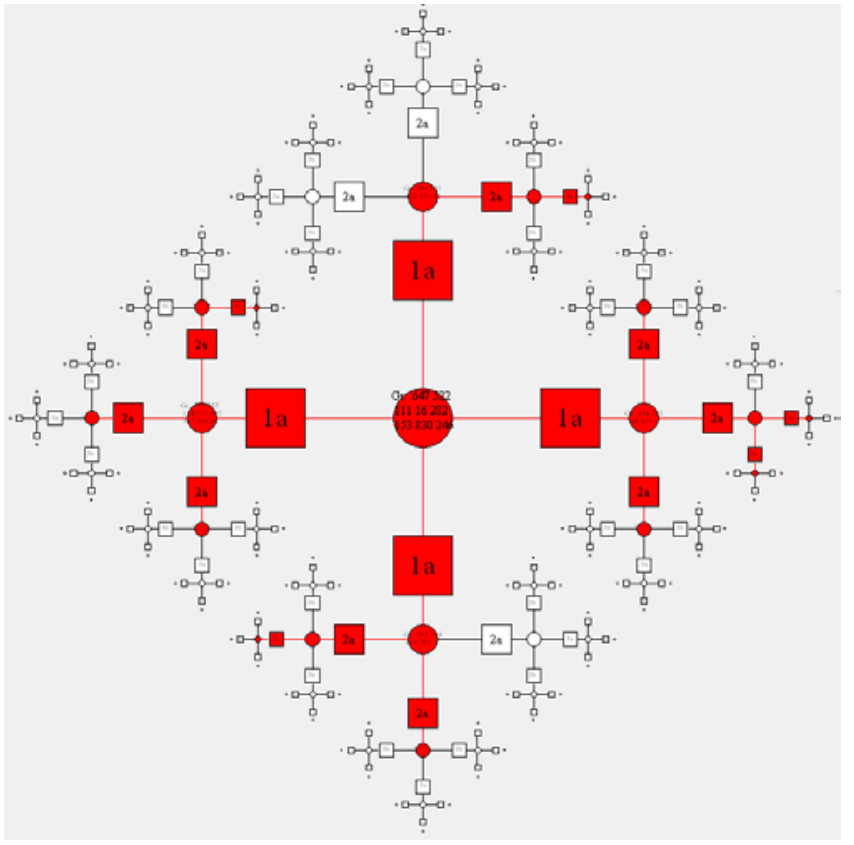

(c)

FIG. 2. Reconstruction of the TRDs in a copper film with ARPGE: (a) Orientation map of the copper grains. (b) Reconstructed TRDs (random colors). (c) Fractal representation of the twinning chain of the large blue TRD (top right of $b$ ). 